\title{
Transmission and reflection in a perfectly amplifying and absorbing medium
}

\author{
P. K. Datta日 \\ Mehta Research Institute of Mathematics and Mathematical Physics, Chhatnag Road, Jhusi, \\ Allahabad-211 019, India
}

\begin{abstract}
We study transmission and reflection properties of a perfectly amplifying as well as absorbing medium analytically by using the tight binding equation. Different expressions for transmittance and reflectance are obtained for even and odd values of the sample length which is the origin of their oscillatory behavior. In a weak amplifying medium, a cross-over length scale exists below which transmittance and reflectance increase exponentially and above which transmittance decays exponentially while the reflectance gets saturated. Depending on amplification transmittance and reflectance show singular behavior at the cross-over length. In a weak absorbing medium we do not find any cross-over length scale. The transmission coefficient behaves similar to that in an amplifying medium in the asymptotic limit. In a strong amplifying/absorbing medium, the transmission coefficient decays exponentially in the large length limit. In both weak as well as strong absorbing media the logarithm of the reflection coefficient shows the same behavior as obtained in an amplifying medium but with opposite sign.
\end{abstract}

PACS Numbers: 42.25.Bs, 71.55.Jv, 72.10-d 
In recent years the study of wave propagation in an active random medium, in the presence of absorption or amplification has attracted much interest [1-23]. Due to the absence of a conservation law for photons, light may be absorbed or amplified in the medium while phase coherence is preserved. The interplay of absorption or amplification and localization has been studied extensively by using the Helmholtz equation with an imaginary dielectric constant of an appropriate sign or by using the Schrödinger equation with imaginary potentials. Experimentally a random amplifying medium can be achieved in a turbid laser dye or a powdered laser crystal [1]. The optical propagation or magnetic excitons in solids which terminate upon reaching trapping sites can be thought of as an absorbing medium. Several results have been obtained in this area. The system plays a dual role as an amplifier or absorber and a reflector [5]. When the strength of the imaginary potential is increased beyond a certain limit both absorber and amplifying scatterer act as a reflector. Absorption suppresses the transmission just as amplification does in the large length limit [6]. A cross-over length scale exists below which the amplification enhances the transmission and above which it reduces the transmission [6, [, 17, 18,. In contrast, the reflectance saturates in the large length limit for both types of media [17, 18]. The distribution of reflectance [8,21], transmittance [5,7] and the phase of transmittance [15] and reflectance [5, 17] for both the active media has been studied by several authors analytically [3, 1, 16,22] as well as numerically [5. 17 [19.

Most of the studies have been made on active random media. For proper understanding of the effect of randomness in active media it is important to study the perfect absorbing as well as amplifying system. But much less attention has been paid in this direction [9, 24, 25]. So, we study here the transmission and reflection properties of both the perfect systems by using the tight binding equation,

$$
\left(E-\epsilon_{n}\right) c_{n}=V\left(c_{n-1}+c_{n+1}\right)
$$

Here, $E$ is the particle energy, $V$ is nearest-neighbor hopping amplitude, $\epsilon_{n}$ is the $n$-th site energy. and $c_{n}$ is the amplitude of the $n$-th site. Without any loss of generality we assume 
$V=1$. For a perfectly active medium $\epsilon_{n}=i \eta$ makes the Hamiltonian non-Hermitian and consequently the particle conservation fails. Here $\eta$ is a fixed real number which is positive (negative) for an absorbing (amplifying) medium. In a previous study 25] it has been shown numerically with some analytical calculations that for a perfectly absorbing medium transmittance decays exponentially with the sample length but reflectance saturates after initial oscillations. On the other hand, for an amplifying medium transmittance initially increases up to a certain length through large oscillations after which it decays and the reflectance also shows an initial increasing behavior after which it saturates to a value larger than unity. In Ref. [9] a perfectly amplifying medium has been studied in the Kronig Penny model. The authors obtain exact expressions for transmission coefficient and the cross-over length. They have also studied the disorder effect on the system. However, we feel that the work on perfect amplifying/absorbing medium is not complete. Some interesting results which may help to understand the properties of active media in the presence of randomness seem to have been overlooked in previous studies [9,24,25]. So, here we perform the analytical study of perfect amplifying as well as absorbing media using Eq. [1. By using the transfer matrix method we obtain exact expressions for the transmission and reflection coefficients for both the media, described by Eq. [. The oscillatory behavior of both transmittance and reflectance is understood from those expressions. Depending on values of $\eta$ for the amplifying medium we find singular behavior in the transmission and reflection coefficients at the cross-over length. For both amplifying and absorbing media, the transmittance and reflectance are studied in different ranges of $\eta$ and sample length.

We now discuss the transfer matrix method [26] to calculate the transmission and reflection coefficients. The active medium consisting of $N$ sites $(n=1$ to $N)$ is placed between two semi-infinite perfect leads with all site-energies are taken to be zero and $V=1$. From Eq. [1 one can easily obtain the site-amplitude for any length of the chain from the initial ones through sequential product of transfer matrices in the following way, 


$$
\left(\begin{array}{l}
c_{N+1} \\
c_{N}
\end{array}\right)=\prod_{i=1}^{N} P_{i}\left(\begin{array}{l}
c_{1} \\
c_{0}
\end{array}\right)
$$

where the transfer matrix

$$
P_{i}=\left(\begin{array}{cc}
E-\epsilon_{i} & -1 \\
1 & 0
\end{array}\right)
$$

Note also that $P_{i}$ is a unimodular matrix. If a plane wave $e^{i k n}$ with energy $E=2 \cos k$ is sent through the perfect lead from one side then the solutions on the two sides of the sample are related by

$$
M=\Omega S^{-1} \prod_{i=1}^{N} P_{i} S
$$

where

$$
\Omega=\left(\begin{array}{cc}
e^{-i k N} & 0 \\
0 & e^{i k N}
\end{array}\right), \quad S=\left(\begin{array}{cc}
e^{i k} & e^{-i k} \\
1 & 1
\end{array}\right) .
$$

The transmission amplitude can be written as

$$
t=\frac{1}{M_{22}}=\frac{-2 i \sin k e^{-i k N}}{(B-C)+\left(A e^{-i k}-D e^{i k}\right)}
$$

and the reflection amplitude

$$
r=-\frac{M_{21}}{M_{22}}=\frac{e^{i k}(D-A)+\left(C e^{2 i k}-B\right)}{(B-C)+\left(A e^{-i k}-D e^{i k}\right)}
$$

where $A, B, C$ and $D$ are four matrix elements of $\prod_{i=1}^{N} P_{i}$. All the four matrix elements have different expressions for even and odd values of $N$ which are given in the table 1 . In our study we set the energy of the incident particle at $E=0$ i.e. at the midband energy. Replacing $A, B, C$ and $D$ in Eq. 3 and 4 we get the exact expression for transmission and reflection coefficients for both even and odd values of $N$ as

$$
\begin{gathered}
T_{\text {even }}=|t|^{2}=\frac{\cosh ^{2} \xi}{(\sinh N \xi+\cosh \xi \cosh N \xi)^{2}} \\
T_{\text {odd }}=\frac{\cosh ^{2} \xi}{(\cosh N \xi+\cosh \xi \sinh N \xi)^{2}}
\end{gathered}
$$




$$
R_{\text {even }}=|r|^{2}=\frac{\sinh ^{2} \xi \sinh ^{2} N \xi}{(\sinh N \xi+\cosh \xi \cosh N \xi)^{2}}
$$

and

$$
R_{\text {odd }}=\frac{\sinh ^{2} \xi \cosh ^{2} N \xi}{(\cosh N \xi+\cosh \xi \sinh N \xi)^{2}}
$$

where $\sinh \xi=\eta / 2$. Subscripts even and odd indicate for even and odd values of $N$ respectively. In Figs. 1-3 we have plotted $\ln T$ and $\ln R$ for weak media as a function of $N$ by using the above equations and from numerical calculations using Eqs. 3 and 4 . It is found that the oscillations arise due to the different behavior of transmission and reflection coefficients for even and odd values of $N$. Using the above equations we next discuss the behavior of transmission and reflection coefficients for both amplifying and absorbing media at different regions of $N$ for both small and large values of $|\eta|$.

We first consider the case of weak amplifying medium i.e. $-1<<\eta<0$ and $N<<$ $N_{\mathrm{cr}}=\frac{4 \ln 2-2 \ln |\eta|}{|\eta|}$. The transmission coefficient $(T)$ shows same behavior for both odd and even values of $N$ as

$$
\ln T \approx|\eta| N
$$

Thus, below $N_{\text {cr }}$ the transmission coefficient increases in the weak amplifying medium exponentially (see Fig. 11) with the rate $|\eta|\left[\right.$ [, 6 ]. For fixed value of $N\left(<<N_{\text {cr }}\right)$ as we increase $|\eta|$ the transmission coefficient increases. This behavior also sustains in the presence of randomness in the medium [18]. The reflection coefficient $(R)$ shows different behavior for even and odd values of $N$ as

$$
\ln R_{\text {even }} \approx 2 \ln \frac{|\eta|}{4}+2 \ln \left(e^{N|\eta|}-1\right)
$$

and

$$
\ln R_{\text {odd }} \approx 2 \ln \frac{|\eta|}{4}+2 \ln \left(e^{N|\eta|}+1\right)
$$

¿From the above two expressions it can be easily understood that the difference between $\ln R_{\text {even }}$ and $\ln R_{\text {odd }}$ is significant for small values of $N$. This is also observed in Fig. 2 where 
the oscillations occur in $\ln R$ (obtained from numerical calculations) for small values of $N$. When $N$ is large but much less than $N_{\text {cr }}$ the reflection coefficient increases exponentially with $N$ with the rate of $2|\eta|$. On the other hand for a fixed value of $N\left(<<N_{\text {cr }}\right)$ as we increase $|\eta|$ both $T$ and $R$ increase. Thus in this limit the amplification enhances the transmission as well as reflection coefficients.

In the asymptotic limit of $N$ (i.e. $N>>N_{\text {cr }}$ ) and for weak amplification the transmission and reflection coefficients do not depend on even and odd values of $N$. They are,

$$
\ln T \approx 4 \ln \frac{4}{|\eta|}-|\eta| N
$$

and

$$
\ln R \approx 2 \ln \frac{4}{|\eta|}
$$

The transmission coefficient decreases exponentially (see Fig. 1) with $N$ at the rate of $|\eta|$. Thus for $N>>N_{\text {cr }}$ amplification suppresses the transmission with the localization length $N_{0}=1 /|\eta|$ [6]. It should be noted that in both the regions $N<<N_{\text {cr }}$ and $N>>N_{\text {cr }}$ the rate of increase and decrease of $T$ is equal. The reflection coefficient attains to a saturation value (see Fig. 2) depending on $|\eta|$. This value increases as $|\eta|$ decreases.

In the case of weak amplification $T$ increases for small values of $N$ and decreases in the asymptotic limit. So, there exists a length scale, called the cross-over length scale where the transmission coefficient shows a maximum. Our next job is to find the cross-over length scale. The transmission coefficient will reach its maximum value when the first derivative of $T$ with respect to $N$ vanishes. From Eq. 5 we find that $T_{\text {even }}$ shows a maximum at

$$
N_{\max }=\frac{1}{2 \xi} \ln \left(\frac{\cosh \xi-1}{\cosh \xi+1}\right) \text {. }
$$

¿From the above expression we find that the positive value of $N_{\max }$ is obtained only when $\eta<0$ i.e. the peak of $T$ occurs in an amplifying medium [6, 7, 9, 18]. On the other hand $T_{\text {odd }}$ goes to infinity as $N \rightarrow N_{\max }$. This is also true for $R_{\text {odd }}$ as the denominator of both $R$ and $T$ is same. The active medium is described here as a discrete system containing $N$ amplifying/absorbing sites where $N$ takes only even and odd integer values. So, with proper choice 
of $\eta$ if $N_{\max }$ becomes odd integer we obtain the singular behavior of transmission coefficient. In Fig. 4 we have plotted the values of $\ln T_{\max }$ (i.e. maximum value of $T$ ) obtained from numerical calculations as a function of $|\eta|$ which contains many sharp peaks indicating the presence of singularity of $T$ at different values of $|\eta|$. This kind of oscillatory behavior is also observed around the peak of the plot of $\ln T_{\max }$ in the presence of randomness in the medium (see Fig.[1] in Ref. [17]). All the singularities in $T$ are destroyed by the randomness in the system. The randomness in the medium suppresses the weak amplification. Consequently, the amplitude of oscillations of $\ln T_{\max }$ decreases with $|\eta|$ decreasing. However, in the perfectly weak amplifying medium the position of the singularities in $T$ are shown in Fig. 5 (obtained from numerical calculations). For comparison we also plot Eq. 14. The deviation occurs at large values of $|\eta|$ due to the discreteness of the system. For any value of $|\eta|$ near $N_{\max }, T_{\text {odd }}$ diverges rapidly to a very large value (infinity, depending upon $\eta$ ) whereas $T_{\text {even }}$ shows a finite maximum value. This indicates that the transmission coefficient oscillates around $N_{\max }$. As the system is discrete we obtain a limit of $\eta$ to get a maximum in $T$. We find that $\eta=-2$ when $N_{\max }=1$. Thus the maximum in $T$ occurs only when $0>\eta>-2$. In the case of weak amplifying medium $(-1<<\eta<0)$ we find $N_{\max }=N_{\mathrm{cr}}$.

For large values of $|\eta|$ (i.e. $|\eta|>>2$ ) and in the asymptotic limit of $N$ the transmission and reflection coefficients show the same behavior for even and odd values of $N$. The transmission coefficient decays exponentially with $N$ as,

$$
\ln T \approx 2 \ln 2+4 /|\eta|+4 /|\eta|^{2}-2\left(\ln |\eta|+1 /|\eta|^{2}\right) N
$$

It should be noted that in the strong amplifying medium the rate of decay is different from that in a weak amplifying medium. The reflection coefficient attains to a saturation value which depends on $\eta$ as

$$
\ln R \approx 4 /|\eta|-8 / 3|\eta|^{3}
$$

Thus as $|\eta|$ increases reflection coefficient in saturation region decreases and approaches to unity. On the other hand $T$ decreases exponentially with $N$. This implies that for larger values of $|\eta|$ the medium behaves as a reflector. 
Now we discuss the transmission and reflection properties for an absorbing medium (i.e. $\eta>0$ ). For weak absorbing medium (i.e. $0<\eta<<1$ ) we find that the expression for the transmission coefficient is the same for even and odd values of $N$ and it decays exponentially [5.19] with $N$ as

$$
\ln T \approx-\eta N .
$$

It should be noted that the rate of decay is the same as in a weak amplifying medium when $N>>N_{\text {cr }}$. To obtain this expression we find that there is a restriction on the sample length of $N>>-N_{\text {cr }}$. This implies that the above expression is valid for any values of $N(>0)$. Hence, like in a weak amplifying medium we do not find any increasing behavior in $\ln T$. Though $T$ does not depend on even and odd values of $N$, the reflection coefficient shows different behavior as,

$$
\ln R_{\mathrm{even}} \approx 2 \ln \frac{\eta}{4}+2 \ln \left(1-e^{-\eta N}\right)
$$

and

$$
\ln R_{\text {odd }} \approx 2 \ln \frac{\eta}{4}+2 \ln \left(1+e^{-\eta N}\right) .
$$

The difference between the two expressions is the last term which is significant for small values of $N$ as long as $e^{-\eta N}$ has significant contributions. For very small values of $N$ (such that $\eta N<<1)$ the last term of $\ln R_{\text {even }}$ increases with $N$ as $\sim 2 \ln (\eta N)-\eta N$ and that of $\ln R_{\text {odd }}$ decreases as $\sim 2 \ln 2-\eta N$. Due to the different behavior of $\ln R$ for small even and odd values of $N$ the oscillatory behavior in $R$ is obtained (see Fig. [3). In asymptotic limit of $N$ we find that the reflection coefficient is independent of $N$ and it increases with $\eta$ as $\sim \eta^{2} / 16$. It should be noted that in the asymptotic limit of $N$ the expression of $\ln R$ for both weak amplifying and absorbing media is the same but with opposite sign.

For large values of $\eta$ (i.e. $\eta>>2$ ) and in the asymptotic limit of $N$ the transmission coefficient is the same for both even and odd values of $N$ and it decays as

$$
\ln T \approx 2 \ln 2-4 / \eta+4 / \eta^{2}-2\left(\ln \eta+1 / \eta^{2}\right) N .
$$


It should be noted that the rate of decay is same as that of a strong amplifying medium (compare Eq. 15 and 20). The reflection coefficient attains to a saturation value which is independent of $N$ as

$$
\ln R \approx-4 / \eta+8 / 3 \eta^{3} .
$$

The expression is again same as that of strong amplifying medium but with opposite sign. It should be noted that in saturation region reflection coefficient increases as $\eta$ increases and in the asymptotic limit of $\eta$ the system behaves like a reflector (as $R \rightarrow 1$ ) [24].

In conclusion, we have studied the transmission and reflection properties of perfectly amplifying and absorbing media by using the tight binding equation. We obtained exact expressions for transmission and reflection coefficients which are different for even and odd values of $N$. This explains the origin of oscillating behavior in $\ln R$ and $\ln T$ in different ranges of $N$. In case of an amplifying medium, a cross-over length scale exists below which $T$ and $R$ increases exponentially and above which $T$ decreases exponentially and $R$ attains to a saturation value. At the cross-over length depending upon values of $\eta$ singular behavior in $T$ and $R$ is obtained. We also found a limit of $\eta$ to get the maximum in $\ln T$. For $|\eta|>>2, T$ decreases exponentially with the rate different from that in a weak amplifying medium and $R$ attains to a saturation value. The expressions of $R$ in the saturation regime are different for weak and strong amplifying media. In case of absorbing media $T$ decays with $N$ like in an amplifying medium in the asymptotic limit of $N$. The logarithm of the reflection coefficient shows the same behavior as in an amplifying medium but with opposite sign. In asymptotic limit of $|\eta|$ and $N$ both absorbing and amplifying medium behaves like reflectors. 


\section{REFERENCES}

a Permanent address: K. N. College, Berhampore, Murshidabad, West Bengal-742 101, India

[1] N. M. Lawandy, R. M. Balachandran, A. SL. Gomes and E. Sauvain, Nature 368, 436 (1994); D. S. Wiersma, M. P. van Albada and Ad Lagendijk, Phys. Rev. Lett. 75, 1739 (1995).

[2] R. L. Weaver, Phys. Rev. B 47, 1077 (1993).

[3] P. Pradhan and N. Kumar, Phys. Rev. B 50, 9644 (1994).

[4] V. Freilikher, M. Pustilnik and I. Yurkevich, Phys. Rev. Lett. 73, 810 (1994)

[5] A. K. Gupta and A. M. Jayannavar, Phys. Rev. B 52, 4156 (1995).

[6] J. C. J. Paasschens, T. Sh. Misipashaev and C. W. J. Beenakker, Phys. Rev. B 54, 11887 (1996).

[7] Z. Q. Zhang, Phys. Rev. B 52, 7960 (1995).

[8] C. W. J. Beenakker, J. C. J. Paasschens and P. W. Brouwer, Phys. Rev. Lett. 76, 1368 (1996).

[9] N. Zekri, H. Bahlouli and A. K. Sen, J. Phys.:Condens. Matter 10, 2405 (1998).

[10] T. Sh. Misirpashaev, J. C. J. Paasschens and C. W. J. Beenakker, Physica A 236, 189 (1997).

[11] M. Yosefin, Europhys. Lett. 25, 675 (1994).

[12] S. John, Phys. Rev. Lett. 53, 2169 (1984).

[13] A. Z. Genack, Phys. Rev. Lett. 58, 2043 (1986).

[14] A. Z. Genack and N. Garcia, Phys. Rev. Lett. 66, 2064 (1991). 
[15] V. Freilikher and M. Pustilnik, Phys. Rev. B 55, R653 (1997).

[16] V. Freilikher, M. Pustilnik, and I. Yurkevich, Phys. Rev. B 56, 5974 (1997).

[17] S. K. Joshi and A. M. Jayannavar, Phys. Rev. B 56, 12038 (1998).

[18] S. K. Joshi and A. M. Jayannavar, cond-mat/9712250.

[19] K. Kim, cond-mat/980334.

[20] W. Deng, D. S. Wiersma, Z. Q. Zhang, Phys. Rev. B 56, 178 (1997).

[21] P. W. Brouwer, Phys. Rev. B 57, 10526 (1998).

[22] J. Heinrichs, Phys. Rev. B 56, 8674 (1997).

[23] A. A. Burkov and A. Yu. Zyuzin, Phys. Rev. B 55, 5736 (1997).

[24] A. M. Jayannavar, Phys. Rev. B 49, 14718 (1994).

[25] A. K. Sen, Mod. Phys. Lett. B 10, 125 (1996).

[26] P. K. Datta, D. Giri and K. Kundu, Phys. Rev. B 47, 10727 (1993). 


\section{FIGURES}

FIG. 1. Plot of $\ln T$ as a function of sample length $N$ for an amplifying medium with $\eta=-0.05$.

FIG. 2. Plot of $\ln R$ as a function of $N$ for the same system as in Fig. 1.

FIG. 3. Same as Fig. 2 but $\eta=0.02$

FIG. 4. Plot of $\ln T_{\max }$ as a function of $|\eta|$ for an amplifying medium.

FIG. 5. Log-log plot of $N_{\max }$ as a function of $|\eta|$ for an amplifying medium. 


\section{TABLES}

TABLE I. The matrix elements of $\prod_{i=1}^{N} P_{i}$ for even and odd values of $N$. Here $\eta=2 \sinh \xi$.

\begin{tabular}{|c|r|r|}
\hline \hline$N$ & Even & Odd \\
\hline Matrix elements & $(-1)^{N / 2 \frac{\cosh (N+1) \xi}{\cosh \xi}}$ & $i(-1)^{(N+1) / 2 \frac{\sinh (N+1) \xi}{\cosh \xi}}$ \\
\hline$A$ & $i(-1)^{(N / 2+1) \frac{\sinh N \xi}{\cosh \xi}}$ & $(-1)^{(N+1) / 2 \frac{\cosh N \xi}{\cosh \xi}}$ \\
\hline$B=-C$ & $(-1)^{N / 2 \frac{\cosh (N-1) \xi}{\cosh \xi}}$ & $i(-1)^{(N+1) / 2 \frac{\sinh (N-1) \xi}{\cosh \xi}}$ \\
\hline$D$ & & \\
\hline \hline
\end{tabular}




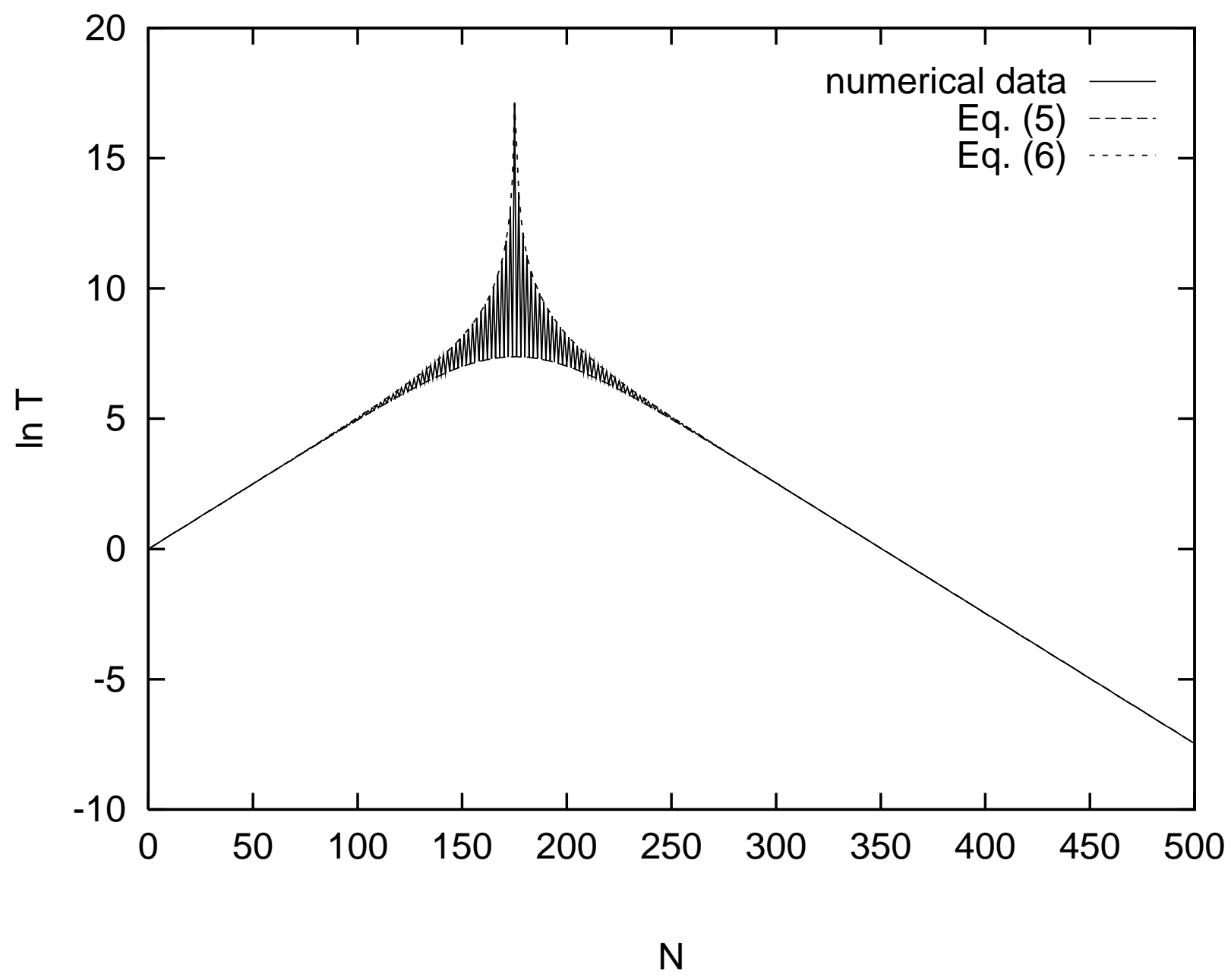

FIG. 1 


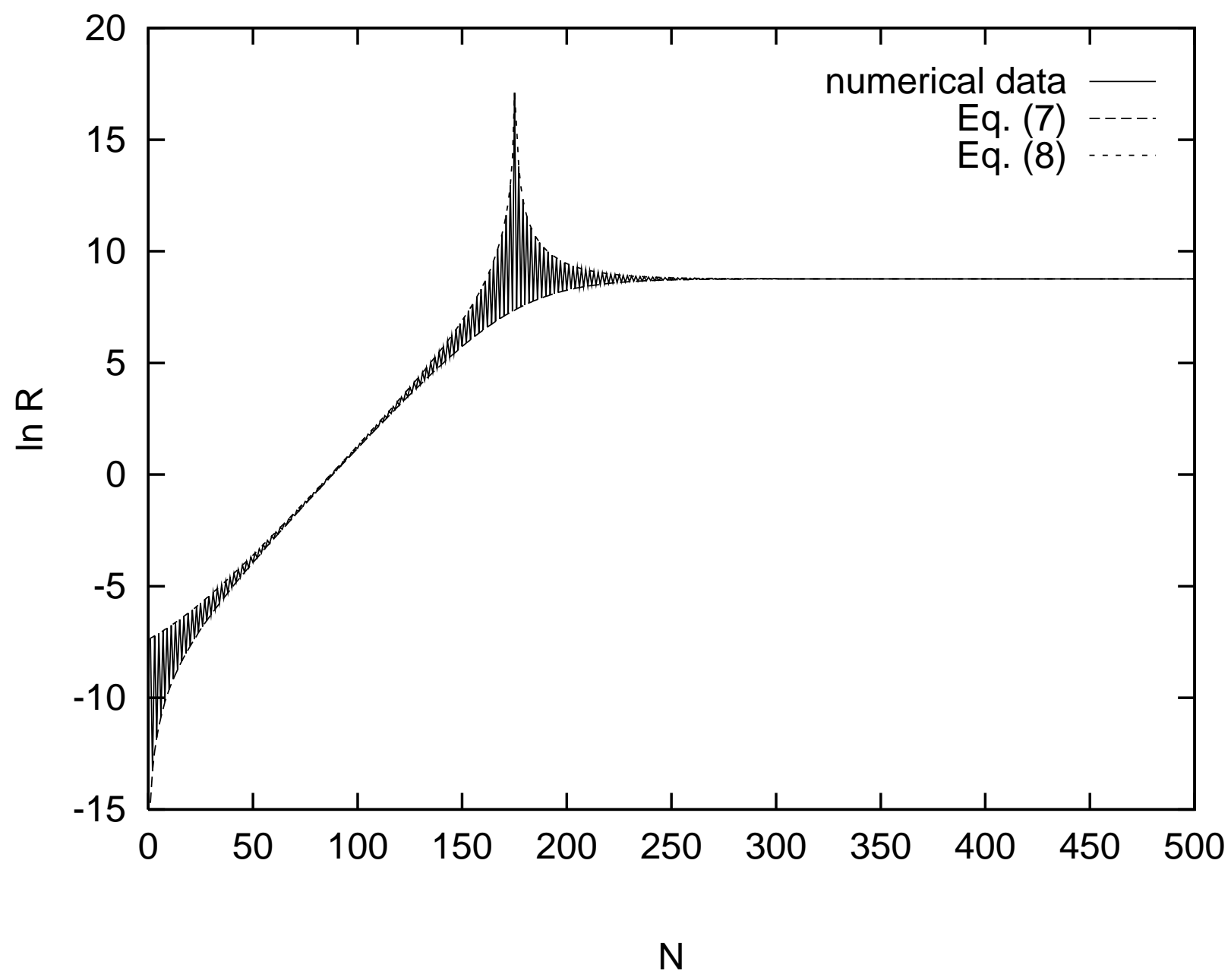

FIG. 2 


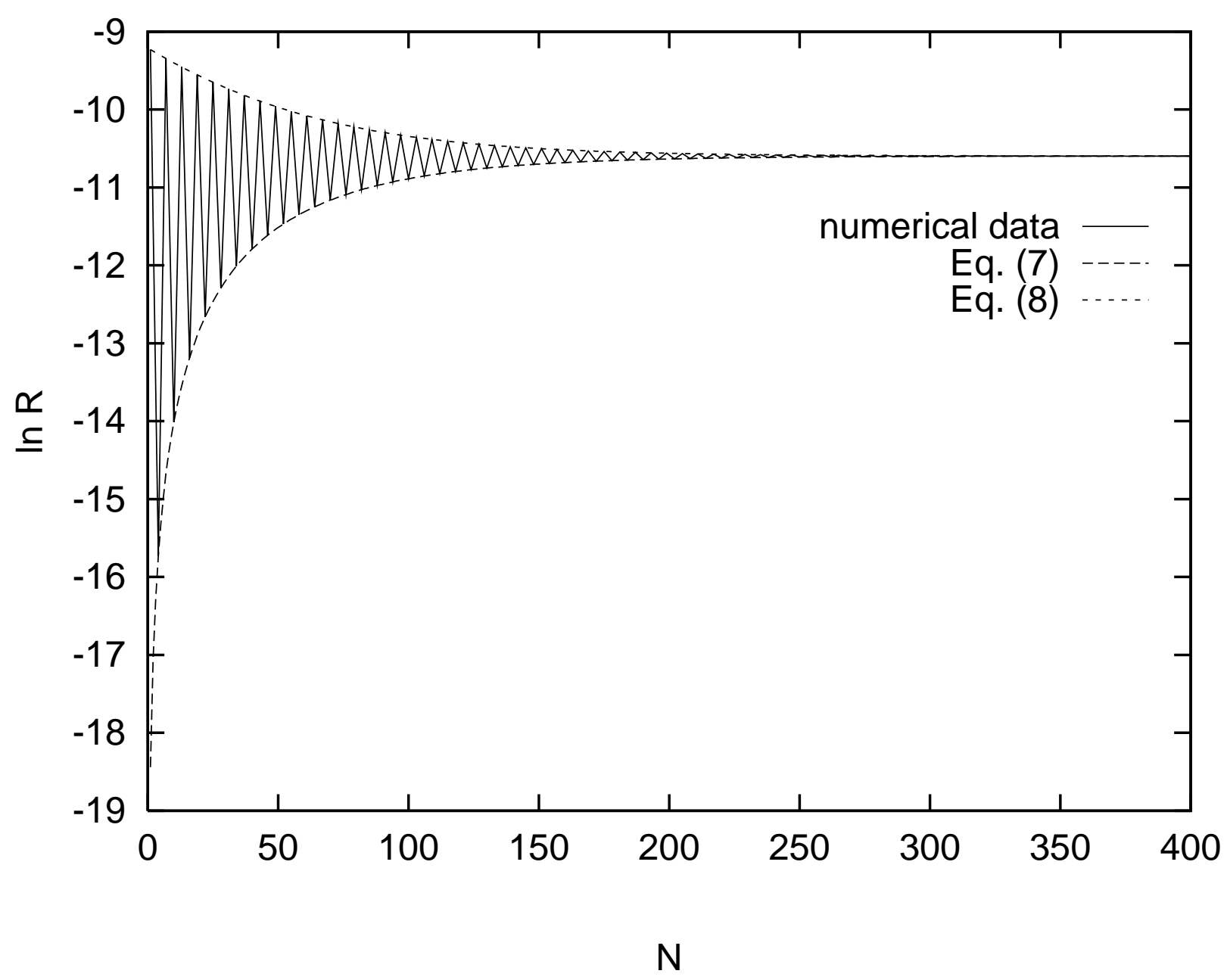

FIG. 3 


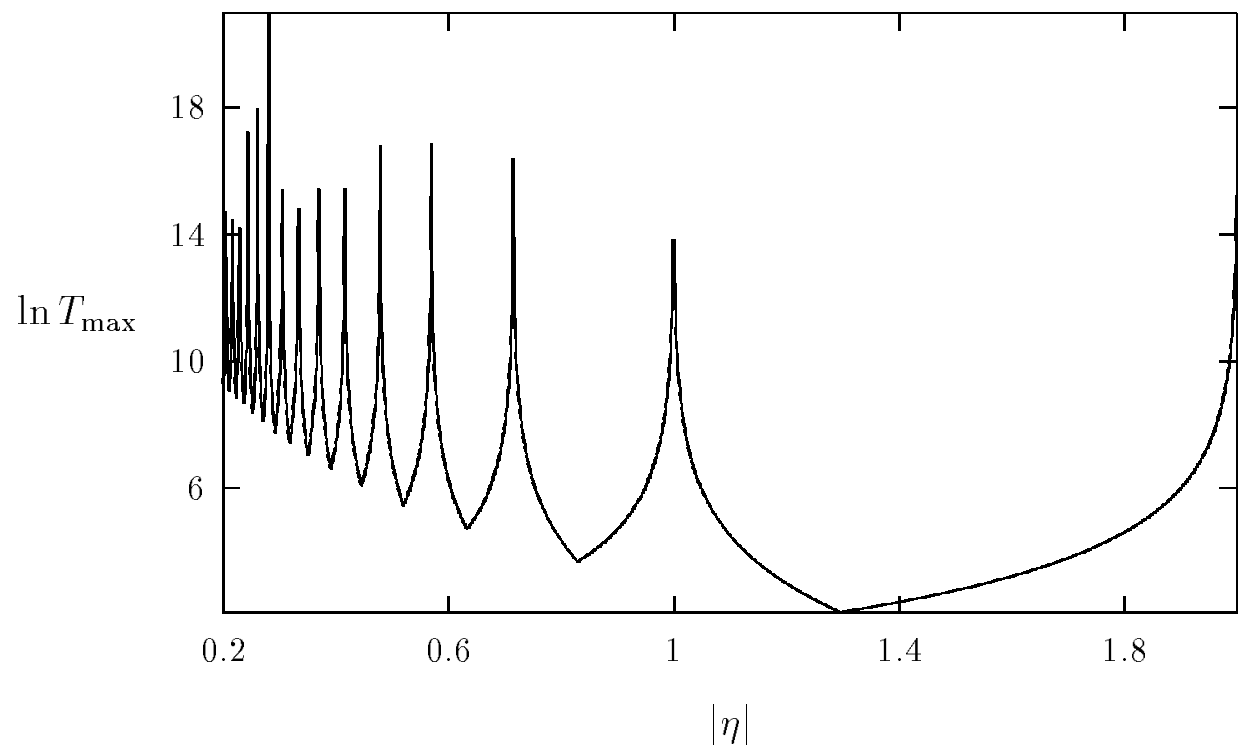

FIG. 4 


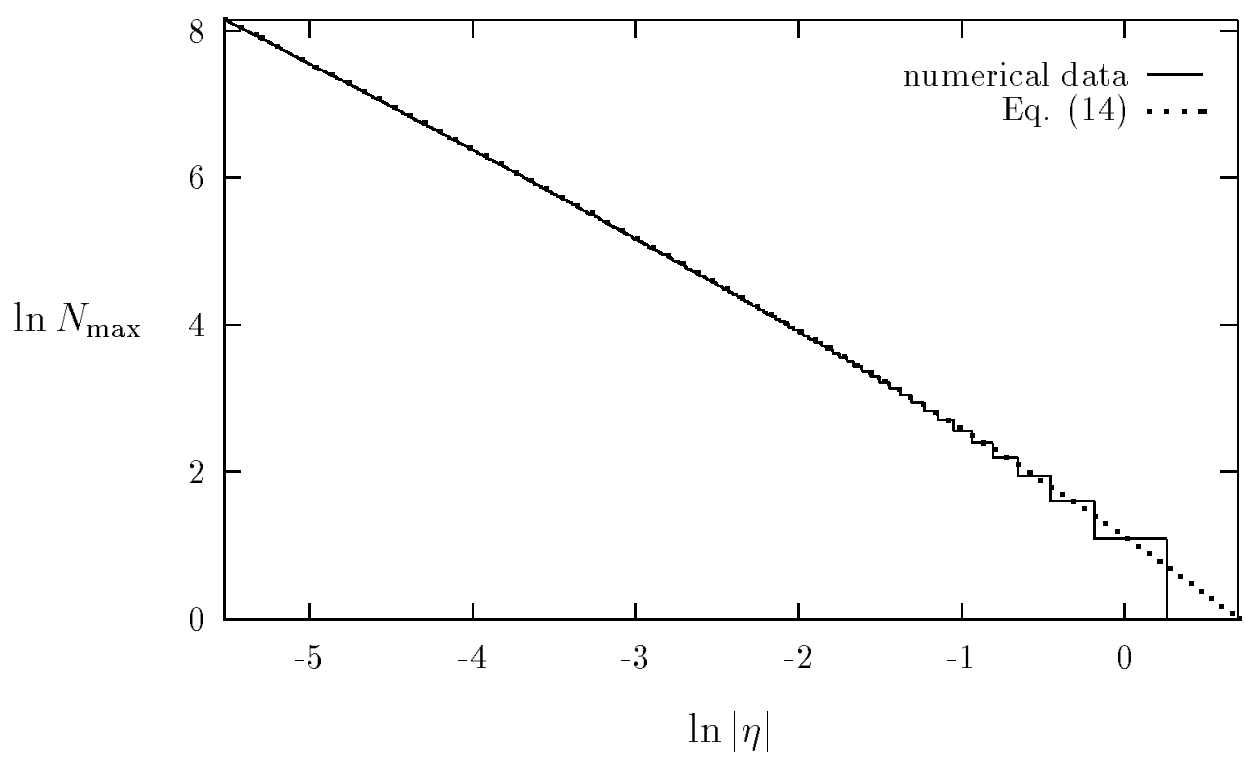

FIG. 5 